\title{
Getting real with wearable data
}

\author{
The outgoing FDA commissioner's comments on the slow integration of data from mobile platforms into clinical \\ research highlight the challenges facing real-world applications of wearables.
}

$\mathrm{n}$ one of Scott Gottlieb's parting shots as

US Food and Drug Administration (FDA)

commissioner, he pointed to the failure of the "clinical research enterprise" to "develop new incentives that reward collaboration and data sharing." The opportunity afforded by "mobile technologies" was, he wrote, being "delayed and stymied" because of "legacy business models that discourage collaboration and data sharing." Gottlieb's FDA recently released a draft framework exploring how real-world data (RWD) from medical claims, electronic medical records (EMRs), mobile platforms and wearable sensors could better inform regulatory decisions. But for wearable devices to be effectively deployed in healthcare and research, the cold truth is that they must overcome not only economic, regulatory and technological challenges but also the obstructive obstinacy of established healthcare culture.

In this issue, a Focus on Wearable Sensors highlights the remarkable progress in 'on-body' devices that can provide continuous physiological measurements of markers in an individual's sweat, tears, saliva or interstitial fluid. Advances in soft electronics and materials, miniaturization and microfluidics, energy harvesting and storage, and wireless communication and smart phone technology are spurring the development of a new generation of sensors that are less intrusive and more reliable. Those sensors hold the promise of gathering personal health data in a continuous manner outside the context of traditional clinical research.

Last December's FDA framework spells out how RWD can be transformed into 'real-world evidence' (RWE)-by better documenting the correlations arising in RWD between the use of a medical product and its clinical benefits or risks, and exploring potential causal relationships. That RWE could support new uses for already approved drugs, or help determine postapproval requirements for drugs, or both. As yet, however, demonstrations of RWE from wearables remain few and far between.

Part of the reason for that is that deploying digital and wearable technology remains largely the province of a few multinational pharmaceutical companies, and, naturally enough, they use it to support their businesses. Health insurance companies do not pay for digital medicine technology because their coverage is based on data on patients and their claims. Healthcare systems, too, do not yet see the value of wearables in clinical practice.

Multinationals like GlaxoSmithKline, Novartis or Lilly have 'chief digital officers' overseeing integration of mobile and wearable technologies into their clinical trial programs. But overall, industry has been slow to integrate wearable technology. In any case, trials are not the real world.

These market issues are compounded by issues related to data collection, hosting, sharing and management. Healthcare systems silos simply cannot handle the continuous stream of RWD coming from wearable devices. Remarkably, according to the American Medical Informatics Association, $75 \%$ of US clinical centers still use fax machines to share EMRs. And while companies like TriNetX and Apigee may address these problems, few are attempting to incorporate longitudinal RWD from wearables with EMRs in a seamless environment (Yale startup Hugo being a notable exception).

In the era of Facebook and Cambridge Analytica and repeated data hacks of EMRs, data privacy and security are also on people's minds. Users may agree implicitly to data sharing when signing up for internet services, but calling this 'informed consent' stretches credulity. Last May, when the European Union's General Data Protection Regulation boosted the protection of individuals' rights over their data, it also created new barriers to data sharing.

Strategies within the nascent direct-toconsumer wearables industry do not help. Although the American Medical Informatics Association has lobbied to update the 1996 US Health Information Portability and Accountability Act to align wearables' data privacy policies with those in healthcare systems, wearables companies are keen to protect their businesses. Consequently, their 'solutions' process fuzzy data through black boxes; their algorithms are proprietary, their data cleanup ill-defined or opaque.

Even if transparency and sharing issues are addressed, wearables need a compelling demonstration of how RWD streaming from a device can be transformed into meaningful RWE. A paper on continuous glucose monitoring published last year provides some pointers.

Conventionally, diabetes patients have their hemoglobin A1c measured every few months when they visit the doctor's office. But the visits are sporadic and intermittent, and rarely coincide with the hypoglycemic (or hyperglycemic) events associated with poorer patient outcomes. In contrast, non-invasive continuous glucose recorders not only provide patients with instantaneous readings on which they can act, but can also provide the physician with weekly summaries detailing how often (e.g., $50 \%$ or $70 \%$ of the time) each patient remains within his or her desired glucose range. By guiding treatment using this 'time in range' - a kind of RWE digital biomarker - continuous glucose data can provide a much more accurate outcome measure than traditional hemoglobin A1c.

The significance of this should not be lost. Healthcare today relies on diagnoses based on binary confirmation of the absence/presence of disease determined at the level of a traditional biomarker taken at a single time point. In the above process, the digital biomarker provides information not only on the presence/absence of disease but also on the trajectory of the disease (and what it looks like in the context of others in the population). How this will be integrated into the provision of drug treatment and patient care remains to be seen-but the FDA under Gottlieb has been committed to adapting regulatory oversight to incorporate it.

Whether Gottlieb's exit from the agency will prompt a rethink seems unlikely. For those who believe that RWE from wearables will improve healthcare regulation and innovation, it is reassuring that one of names in the frame as a permanent replacement for Gottlieb is Amy Abernethy. Abernethy is currently deputy commissioner of the FDA and previously chief medical officer and chief scientific officer at the pioneering EMR mining company Flatiron Health.

Published online: 2 April 2019 https://doi.org/10.1038/s41587-019-0109-z 